\title{
Epidemiology of neonatal stroke: A population-based study
}

\author{
Breanna Clive $\mathbf{M D}^{1}$, Michael Vincer $\mathbf{M D}^{1}$, Tahani Ahmad $\mathbf{M D}^{2}$, Naeem Khan $\mathbf{M D}^{2}$, Jehier Afifi $\mathbf{M D}^{1}$, \\ Walid El-Naggar MD $^{1}$
}

${ }^{1}$ Department of Pediatrics, Dalhousie University, Halifax, Nova Scotia; ${ }^{2}$ Departemnt of Diagnostic Imaging, Dalhousie

University, Halifax, Nova Scotia

Correspondence: Breanna Clive, IWK Health Centre, Dalhousie University, 5850/5980 University Ave, PO Box 9700, Halifax,

Nova Scotia B3K 6R8. Telephone (778)-694-9403, fax (902)-470-7542, e-mail bclive@alumni.ubc.ca

\begin{abstract}
Objective: The goal of this study was to obtain population-based data on the incidence, clinical presentation, management, imaging features, and long-term outcomes of patients with all types of neonatal stroke (NS).

Methods: Full-term neonates with NS born between January 2007 and December 2013 were identified through the Nova Scotia Provincial Perinatal Follow-up Program Database. Perinatal data and neonatal course were reviewed. Neurodevelopmental outcomes were assessed at 18 and 36 months of age using standardized testing.

Results: Twenty-nine neonates with NS were identified during the study period, giving an incidence of 47 per 100,000 live births in Nova Scotia. Arterial ischemic stroke was the most common stroke type $(76 \%)$, followed by neonatal hemorrhagic stroke (17\%), then cerebral sinovenous thrombosis (7\%). The majority of neonates presented with seizures (86\%) on the first day of life (76\%). At 36 months of age, $23(79 \%)$ of the children had a normal outcome, while $3(10 \%)$ were diagnosed with cerebral palsy ( 2 with neonatal arterial stroke and one with neonatal hemorrhagic stroke) and 3 (10\%) had recurrent seizures ( 1 patient from each stroke subtype group).

Conclusion: The incidence of NS in Nova Scotia is higher than what has been reported internationally in the literature. However, the neurodevelopmental outcomes at 3 years of age are better. Further studies are required to better understand the reasons for these findings.
\end{abstract}

Keywords: Cerebral palsy; Neonatal stroke; Stroke.

Neonatal stroke (NS), a major cause of hemiparetic cerebral palsy, is defined as a cerebrovascular incident causing interruption of blood flow that presents in the first 28 days postnatally and is confirmed by neuroimaging or pathological studies (1). The condition has been reported mainly in term neonates with an incidence of approximately 25 in 100,000 term live births $(2,3)$. NS can be further classified as neonatal arterial ischemic stroke (NAIS), neonatal hemorrhagic stroke (NHS), or cerebral sinovenous thrombosis (CSVT). The majority of the literature is focused on NAIS, however, each of these entities is discrete in terms of the typical clinical presentations and outcomes (2). The pathophysiology of NS is not well understood, but it is believed to be multifactorial with maternal, perinatal, and neonatal factors contributing. A common associated neonatal risk factor for NAIS is the presence of congenital cardiac disease (1).

Regardless of stroke type, NS most often presents with seizures in the early neonatal period and over half of the affected children will have long-term neurological disabilities (4). Motor deficits have been reported to occur in about 50 to $60 \%$ of the cases, with hemiplegic cerebral palsy being the most common outcome. Other neurodevelopmental problems include recurrent seizures, cognitive disabilities, and behavioural disorders $(4,5)$. 
Population-based studies assessing NS, especially CSVT and NHS, using standardized follow-up methods are rare. We aimed to obtain recent population-based data on the incidence, clinical features, and long-term outcomes of patients with all types of symptomatic NS. Our study is unique in that our entire population is evaluated at the same tertiary care centre and follow-up methods are standardized. We hope this new information will lead to a better understanding of the epidemiology of NS and improve both counselling of families and long-term outcomes.

\section{SUBJECTS AND METHODS}

\section{Study population and data collection}

In this retrospective study, full-term neonates (gestational age $\geq 37$ weeks) born between January 2007 and December 2013 to mothers living in Nova Scotia and diagnosed with symptomatic NS by brain magnetic resonance imaging (MRI) at the IWK Health Centre, were identified if categorized as 'neonatal stroke' in the province's Perinatal Follow Up Program database. This database includes data on neonates born in Nova Scotia with many different diagnoses, such as prematurity and various brain injuries. With parental consent, infants with NS are enrolled in this database at the time of diagnosis at the IWK Health Centre. Associated demographic and perinatal data are collected at the time of enrolment, while follow-up data are collected prospectively. Additional required data was obtained directly from hospital records. In this study, infants with chromosomal or major congenital anomalies, including significant cardiac lesions, and those with evidence of hypoxic ischemic encephalopathy (HIE) were excluded. The Research Ethics Board at the IWK Health Centre approved this study.

\section{Neuroimaging}

All patients had brain MRI studies performed at the IWK Health Centre using a standardized neonatal brain MRI protocol (6). This included axial, T1- and T2-weighted, diffusion-weighted imaging (DWI), axial susceptibility-weighted imaging (SWAN), sagittal T1 flair and coronal T2-weighted imaging of the brain in addition to 3D-Time-of-Flight (TOF) magnetic resonance angiography (MRA), and venography (MRV). The studies were performed within 24 to 72 hours of presentation. Two masked paediatric neuroradiologists re-evaluated the MRI studies in two separate sittings.

NS was classified as NAIS if the distribution of the parenchymal signal abnormality on T1, T2, and DWI was confined to a known arterial territory. Strokes were classified as NHS if the predominant signal intensity was blood. These were further classified as primary if there was no underlying vascular lesion or bleeding diathesis to explain the bleed. Any arterial ischemic changes with secondary bleeding were characterized as NAIS with secondary hemorrhagic transformation, not primary NHS. CSVT was identified if there was obvious venous thrombosis on the standard sequences and/or if MRV had parenchymal changes along the distribution of a venous drainage area. There is no standard definition of stroke size in the literature for neonates. An arbitrary classification scheme was used to describe stroke size in this study and was adapted to all types of stroke. NAIS and CVST timing were determined using the known varying signal intensities of different sequences, including DWI in the evolution of these disorders $(6,7)$. The timing of NHS was assigned based on the predicted evolution of blood signal on MRI within the affected parenchyma (8) (Definitions for size and timing provided in Table 2).

\section{Outcomes}

Long-term outcome data were obtained from the Perinatal Follow Up Program database. Through this program, patients have a thorough history, physical and neurological exam, and developmental screening at multiple time points including at 18 and 36 months of age. The Cognitive Adaptive Test/Clinical Linguistic and Auditory Milestone Scale (CAT/CLAMS) (9) and the Bayley Scales of Infant and Toddler Development-III (Bayley-III) (10), common validated and standardized developmental assessment tools, were used at 18 and 36 months of age, respectively, to assess neurodevelopmental outcomes. A CAT/CLAMS score of $<83$ was considered predictive of significant neurodevelopmental impairment (NDI) at 36 months of age (11). On the Bayley-III, a mental development index (MDI) score of $<85$ was defined as indicative of NDI and a MDI $<70$ was classified as significant NDI (12). Cerebral palsy was diagnosed based on physical examination and neurologic assessment performed by physiotherapists and physicians using the Gross Motor Functional Classification System (GMFCS). Recurrent seizure data were obtained from medical records.

\section{Statistical analysis}

The number of total live births in Nova Scotia from January 2007 to December 2013 was obtained from Statistics Canada and used to calculate the incidence of NS. The results regarding patient characteristics, clinical course, MRI features, and outcomes were presented using descriptive statistics. Mean (standard deviation), median (interquartile range), and relative frequency, respectively, were used to describe clinical characteristics as appropriate.

\section{RESULTS}

\section{Incidence and patient population}

There were 33 patients identified with NS during the study period. Of these, two patients had multiple congenital anomalies, including structural heart lesions, and two had HIE. Therefore, there were a total of 29 neonates included (41\% male) with a median gestational age of 39 weeks (range 37 to 42 weeks) and mean birthweight of 3,279 $\pm 569 \mathrm{~g}$. All patients 
were products of singleton gestations. As reported by Statistics Canada (13), there were 61,991 total live births in Nova Scotia from 2007 to the end of 2013, giving an incidence of NS of 47 per 100,000 live births (95\% confidence interval [CI]: 33 to 67 ). There were 22 (26\%) neonates with NAIS, 5 (17\%) with NHS, and 2 (7\%) with CSVT. Therefore, the incidence of NAIS was 35 per 100,000 live births (95\% CI: 23 to 54), NHS 8 per 100,000 live births (95\% CI: 3 to 19), and CSVT 3 per 100,000 live births ( $95 \% \mathrm{CI}: 1$ to 12 ). There was a trend toward increasing incidence over time.

\section{Clinical presentation and course in NICU}

The majority of infants presented with seizure (86\%) in the first 24 hours of life (76\%, range 0.2 to 528 hours) and in $69 \%$ of neonates, it was the only presenting sign. Figure 1 shows the presenting signs for infants with NAIS. Of the neonates with NHS, three presented with seizures, one had an apneic spell, and another had increasing head circumference and irritability. Both neonates with CSVT presented with seizure. One infant with NAIS presented with intrauterine growth restriction and an abnormal cranial ultrasound prompting MRI evaluation. The median length of hospital stay was 7 days (range 4 to 21 days). Further details of the clinical course and management (based on physician discretion) for infants with NAIS can be found in Table 1 .

\section{Neuroimaging}

The majority of patients had NAIS with 17 (77\%) of these in the middle cerebral artery territory. The posterior cerebral artery was involved in eight (36\%) patients with NAIS and the anterior cerebral artery in five patients (23\%). Greater than one territory was affected in six (27\%) neonates and greater than two territories were involved in two (9\%) patients with NAIS. There were no cases of intraventricular hemorrhage. There were three cases of secondary hemorrhagic transformation in areas of ischemia and these were classified as NAIS, not NHS. One CSVT occurred in a superficial cortical vein and the other in

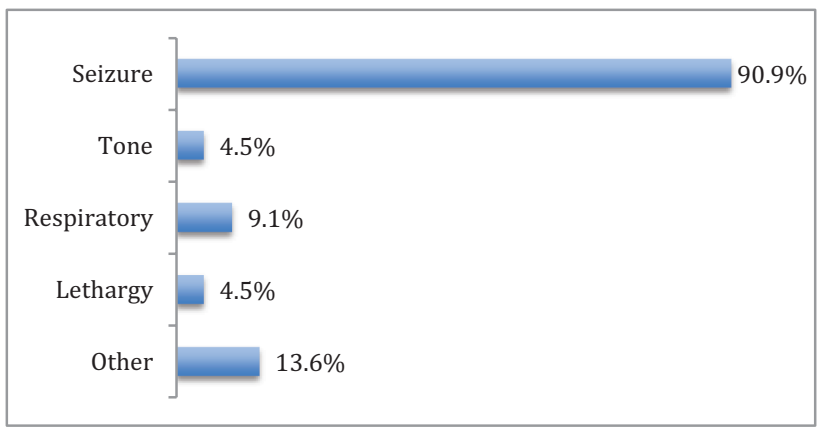

Figure 1. Clinical presenting signs of neonates with neonatal ischemic stroke. The majority of patients presented with seizures. Some patients demonstrated more than one presenting sign. the sigmoid sinus. No patients had ventriculomegaly or positive MRA. Of the patients who developed cerebral palsy, one had NHS with involvement of the basal ganglia while the other two had NAIS (one with involvement of the right frontal lobe and the other was widespread, involving the basal ganglia, internal capsule, corticospinal tract and all lobes of the brain). See Table 2 for additional neuroimaging details.

\section{Long-term outcomes}

No patients with NS died within the study period. Of the 29 patients with NS, 28 (97\%) were assessed in the Perinatal Follow-Up Program clinic at 18 and 36 months. All patients had normal neurodevelopmental assessments except for three patients diagnosed with cerebral palsy (two with NAIS and one with NHS) and three different patients with recurrent seizures (one patient from each stroke type group, Table 3). Two of the children with cerebral palsy had CAT/CLAMS predictive of significant NDI. Their Bayley-III motor scores were both between 70 and 85 in keeping with NDI. The third patient received their assessment in neurology clinic and received a diagnosis of cerebral palsy based on the same criteria as the PFUP, but did not have associated CAT/CLAMS and BayleyIII testing completed.

\section{DISCUSSION}

In this population-based study, we identified the incidence of NS to be 47 per 100,000 live births with NAIS accounting for 35 per 100,000 live births, which is higher than previously reported. While the clinical presentation and neuroimaging findings are in keeping with previous studies, the long-term neurodevelopmental outcomes are better.

Estimation of the true incidence of NS is difficult as comparison of studies is limited by variability in study methods, inclusion criteria, and the types of NS assessed. Studies assessing all types of stroke in a single population are rare. The overall incidence of NS is often cited as 25 per 100,000 live births (2,3). In keeping with previous reports, the most common type of NS in our study was NAIS, but our incidence of 35 per 100,000 live births is higher. Review of a number of population-based studies provides an incidence of NAIS anywhere from 5 to 18 per 100,000 live births $(2,5,14-16)$ with the majority of these studies also including preterm infants. Hospital-based studies have estimated the incidence of CSVT to be 3 to 12 per 100,000 live births $(17,18)$ and NHS to be 6 to 11 per 100,000 live births $(19,20)$. Our incidences of CSVT and NHS are similar to the literature.

The province of Nova Scotia is unique in that all neonates requiring a brain MRI are referred to the same tertiary care centre. The higher NAIS incidence in our study may be a more accurate estimation as diagnosis and standardized follow-up occurs in a single centre, allowing for more complete 
Table 1. Clinical course for NAIS in the neonatal intensive care unit

\begin{tabular}{|c|c|c|}
\hline Consultations & $\mathrm{N}(\%)$ & \\
\hline Neurology & $21(95)$ & \\
\hline Cardiology & $13(59)$ & \\
\hline Hematology & $12(54)$ & \\
\hline Ophthalmology & $4(18)$ & \\
\hline Investigations & Performed, N (\%) & Results abnormal, N (\%) \\
\hline Blood culture ${ }^{b}$ & $19(86)$ & $2(9)$ \\
\hline Lumbar puncture $^{\mathrm{b}}$ & $15(68)$ & $1(5)$ \\
\hline Echocardiogram & $12(55)$ & $4(18)^{a}$ \\
\hline Basic coagulation studies $^{c}$ & $8(36)$ & $0(0)$ \\
\hline Advanced coagulation studies ${ }^{c}$ & $7(32)$ & $1(5)^{b}$ \\
\hline Newborn Hearing Screen & $21(95)$ & $0(0)$ \\
\hline Treatment & $\mathrm{N}(\%)$ & \\
\hline Anticoagulation & $0(0)$ & \\
\hline Antibiotics & $15(68)$ & \\
\hline Anticonvulsants short course ${ }^{d}$ & $20(91)$ & \\
\hline Anticonvulsants long course $\mathrm{e}^{\mathrm{d}}$ & $6(27)$ & \\
\hline
\end{tabular}

${ }^{a}$ Abnormalities included: two patent foramen ovales and two small ventricular septal defects. ${ }^{b}$ Abnormalities = positive cultures felt not to be the cause of stroke/seizure. 'Basic coagulation studies included INR, PT, and PTT. Advanced coagulation studies included at least one of protein C and S, antithrombin III, factor V Leiden, antiphospholipid antibodies, factor VIII, plasma homocysteine and prothrombin. One patient was

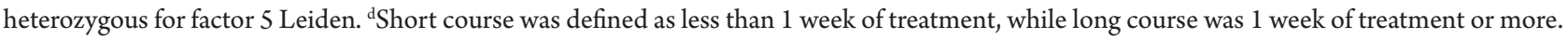

data collection. This study therefore represents a true population-based sample. It is possible that our study, as well as other studies, have underestimated the incidence due to missed cases with subtle presentations. In addition, any infants with suspected NS who died before the diagnosis could be confirmed with MRI would not be included in our study. We noticed a trend toward increasing incidence over the years in our population, which may be a true finding or may be attributed to more awareness, lower threshold for referral, and/or more MRI imaging over the years.

Similar to other studies, we found that left-sided strokes were more common $(15,21,22)$. It is suspected that NAIS arises from emboli in the placenta that pass through the patent foramen ovale into the ascending aorta, where the path of least resistance following that is the left common carotid artery (23). Unfortunately, due to our small sample size, we were unable to correlate stroke location or size with outcome. For example, it has been shown that large stroke size and involvement of the basal ganglia or internal capsule can lead to poorer outcomes $(5,24,25)$. While some of these structures were affected in our neonates with cerebral palsy, there were also many infants with normal outcomes who also had these areas affected and overall sample size was too small to make any statistical correlations.

In our population, none of those who received an echocardiogram had results to explain the cause of stroke. While two patients were excluded for major congenital heart disease, these patients had obvious signs of cyanotic heart lesions shortly after birth. In neonates without cardiac instability, the yield of an echocardiogram was low. Coagulation studies, although the types and timing was variable, also had low yield, with no major thrombophilia conditions identified that could explain the cause of stroke. Two case-control studies have shown no difference in thrombophilia genes or coagulation factors between healthy neonates and those with NS $(26,27)$. Better understanding of the causality and frequency of risk factors could help to create a more standardized approach to investigations in the NICU.

Another significant finding in our study is the better neurodevelopmental outcomes of NAIS patients as only $9 \%$ were diagnosed with cerebral palsy at 36 months of age. This rate is significantly lower than the rates ranging from 26 to $68 \%$ previously reported in the literature $(5,15,22)$. In addition, only $5 \%$ of our NAIS patients had recurrent seizures or epilepsy, whereas other studies have reported rates of 23 to $37 \%(5,25)$. These studies all excluded preterm infants and those with HIE, except the study by Grunt et al., which did include three infants with HIE, but this would not account fully for the higher incidence of cerebral palsy observed (15). While Wusthoff et al. included infants with congenital cardiac disease in her study, recalculated results excluding these patients still shows a higher incidence of seizures at $24 \%$ (25). Our study also found no significant cognitive deficits on the Bayley-III, similar to other studies, however 
Table 2. Brain MRI characteristics of patients with neonatal stroke

\begin{tabular}{|c|c|c|c|}
\hline Stroke type & NAIS (\%) & NHS (\%) & $\operatorname{CSVT}(\%)$ \\
\hline $\mathrm{N}$ & $22(76)$ & $5(17)$ & $2(7)$ \\
\hline \multicolumn{4}{|l|}{$\operatorname{Size}^{a}$} \\
\hline Small & $8(36)$ & $4(80)$ & $1(50)$ \\
\hline Moderate & $9(41)$ & $0(0)$ & $1(50)$ \\
\hline Large & $5(23)$ & $1(20)$ & $0(0)$ \\
\hline \multicolumn{4}{|l|}{ Side } \\
\hline Left & $13(59)$ & $4(80)$ & $1(50)$ \\
\hline Right & $5(23)$ & $0(0)$ & $0(0)$ \\
\hline Bilateral & $4(18)$ & $1(20)$ & $1(50)$ \\
\hline \multicolumn{4}{|l|}{ Structures Involved ${ }^{b}$} \\
\hline Cortex & $20(91)$ & $2(40)$ & $1(50)$ \\
\hline Basal ganglia & $10(45)$ & $2(40)$ & $0(0)$ \\
\hline Internal capsule & $8(36)$ & $0(0)$ & $0(0)$ \\
\hline Thalamus & $7(32)$ & $0(0)$ & $0(0)$ \\
\hline Corticospinal tract & $8(36)$ & $0(0)$ & $1(50)$ \\
\hline Frontal lobe & $15(68)$ & $1(20)$ & $1(50)$ \\
\hline Temporal lobe & $7(32)$ & $0(0)$ & $1(50)$ \\
\hline Parietal lobe & $9(41)$ & $3(60)$ & $1(50)$ \\
\hline Occipital lobe & $4(18)$ & $1(20)$ & $1(50)$ \\
\hline Periventrcular & $6(27)$ & $1(20)$ & $0(0)$ \\
\hline \multicolumn{4}{|l|}{ Timing $^{c}$} \\
\hline Acute & $20(90)$ & $1(20)$ & $2(100)$ \\
\hline Subacute & $1(5)$ & $3(60)$ & $0(0)$ \\
\hline Chronic & $1(5)$ & $1(20)$ & $0(0)$ \\
\hline
\end{tabular}

ACA Anterior cerebral artery; CSVT Cerebral sinovenous thrombosis; MCA Middle cerebral artery; NHS Neonatal hemorrhagic stroke; PCA Posterior cerebral artery.

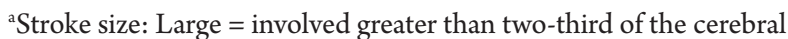
hemisphere, Moderate $=$ involved between one-third and two-third of the cerebral hemisphere, Small = involved less than one-third of the cerebral hemisphere. ${ }^{b}$ Some infants had strokes in more than one area. ${ }^{\mathrm{c}}$ Acute $=$ less than 7 days, Subacute $=1-4$ weeks, Late $=$ greater than 4 weeks.

longer-term studies assessing school-age children have shown deficits $(28,29)$. Population-based studies of CSVT and NHS are limited. A recent study by Cole et al. showed poor neurological outcome in $40 \%$ of patients with idiopathic NHS (20). Epilepsy rates in NHS have been shown to range from 6 to $9 \%(20,30)$. Patients with CSVT tend to have worse outcomes with neurological impairment present in 67 to $79 \%$ of survivors $(31,32)$.

The improved neurodevelopmental outcomes in our study population are promising. Many of the above-referenced studies were carried out close to 10 years ago, and it is possible that management and early detection of these neonates have improved over the years leading to better outcomes. Our follow-up program also provides excellent support and early intervention to patients and may have helped to enhance outcomes.
Table 3. Outcomes of patients with neonatal stroke

\begin{tabular}{lccc}
\hline 18-month follow-up & NAIS & NHS & CSVT \\
\hline N & 21 & 5 & 2 \\
CAT /CLAMS & $107(14)$ & $93(18)$ & $115(4)$ \\
Average Score ( \pm SD) & & & \\
Score <83 N (\%) & $1(5)$ & $1(20)$ & $0(0)$ \\
\hline 36-month follow-up & NAIS & NHS & CSVT
\end{tabular}

\section{Motor}

Number Tested

$\begin{array}{lll}18 & 5 & 2\end{array}$

Bayley-III Motor

$103(10) \quad 98(9) \quad 97(8)$

Average Scores $( \pm \mathrm{SD})$

Significant NDI N (\%) $\quad 0(0) \quad 0(0) \quad 0(0)$

NDI N (\%)

$1(6) \quad 1(20) \quad 0(0)$

Cognitive

Number Tested

20

Bayley-III Cognitive

Average Scores $( \pm$ SD)

Significant NDI N (\%)

$103(9)$

(9)

4

$96(5)$

2

NDI N (\%)

$\begin{array}{lll}0(0) & 0(0) & 0(0) \\ 0(0) & 0(0) & 0(0)\end{array}$

Language

Number Tested

Bayley-III Language

Average Scores $( \pm \mathrm{SD})$

Significant NDI N (\%)

$\begin{array}{ccr}18 & 3 & 1 \\ 110(13) & 93(9) & 118\end{array}$

NDI N (\%)

$0(0) \quad 0(0) \quad 0(0)$

Other Outcomes N (\%)

Number Tested

$0(0) \quad 0(0)$

$0(0)$

Cerebral palsy

22

Recurrent seizures

Recurrent stroke

Blindness

2

2 (9)

5

2

1 (5)

1 (20)

$0(0)$

$1(5)$

1 (20)

$1(50)$

$0(0)$

$0(0)$

$0(0)$

$0(0)$

$0(0)$

$0(0)$

Deafness

$0(0)$

$0(0)$

$0(0)$

Definitions: CAT/CLAMS Score $<83=$ Predictive of significant NDI. Significant NDI $=$ MDI $<70 \%$. NDI $=$ MDI $<85 \%$. Percentages represent the number of positive results per patients tested.

Bayley-III Bayley Scales of Infant and Toddler Development; CAT/CLAMS Cognitive Adaptive Test/Clinical Linguistic and Auditory Milestone Scale; MDI Mental developmental index; NDI Neurodevelopmental impairment.

The ability of the young brain to adapt to injury was described as early as 1936 by Kennard and this 'neuroplasticity' is a key process allowing for neonates to have improved outcomes from stroke compared to adult patients. There are exciting treatments on the horizon to 'repair' the injured brain, including noninvasive brain stimulation and stem cell therapy that may lead to further improvement in outcomes $(33,34)$.

In conclusion, the incidence of NAIS in Nova Scotia is higher than what has been reported in other population-based 
studies. The majority of our patients had good neurodevelopmental outcomes at 36 months of age. There is more to learn about the different stroke types, possible preventative and therapeutic strategies, and prediction of outcomes in these children.

\section{ACKNOWLEDGEMENTS}

The authors would like to acknowledge Dr. Stefan Kuhle for his contribution to this study.

Funding Information: There are no funders to report for this submission. Potential Conflicts of Interest: All authors: No reported conflicts of interest. All authors have submitted the ICMJE Form for Disclosure of Potential Conflicts of Interest. Conflicts that the editors consider relevant to the content of the manuscript have been disclosed.

\section{References}

1. Rutherford MA, Ramenghi LA, Cowan FM. Neonatal stroke. Arch Dis Child Fetal Neonatal Ed 2012;97(5):F377-84

2. Lynch JK, Nelson KB. Epidemiology of perinatal stroke. Curr Opin Pediatr 2001;13(6):499-505

3. Raju TN, Nelson KB, Ferriero D, Lynch JK; NICHD-NINDS Perinatal Stroke Workshop Participants. Ischemic perinatal stroke: Summary of a workshop sponsored by the national institute of child health and human development and the national institute of neurological disorders and stroke. Pediatrics 2007;120(3):609-16.

4. Kirton A, Deveber G. Life after perinatal stroke. Stroke 2013;44(11):3265-71.

5. Lee J, Croen LA, Lindan C, et al. Predictors of outcome in perinatal arterial stroke: A population-based study. Ann Neurol 2005;58(2):303-8.

6. Lequin $\mathrm{MH}$, Dudink J, Tong KA, Obenaus A. Magnetic resonance imaging in neonatal stroke. Semin Fetal Neonatal Med 2009;14(5):299-310.

7. Walecki J, Mruk B, Nawrocka-Laskus E, Piliszek A, Przelaskowski A, Sklinda K. Neuroimaging of cerebral venous thrombosis (CVT) - old dilemma and the new diagnostic methods. Pol J Radiol 2015;80:368-73.

8. Kidwell CS, Wintermark M. Imaging of intracranial haemorrhage. Lancet Neurol $2008 ; 7(3): 256-67$

9. Wachtel RC, Shapiro BK, Palmer FB, Allen MC, Capute AJ. CAT/CLAMS. A too for the pediatric evaluation of infants and young children with developmental delay. Clinical adaptive test/clinical linguistic and auditory milestone scale. Clin Pediatr (Phila) 1994;33(7):410-5

10. Bayley N. Manual for the Bayley scales of infant and toddler development. 3rd ed. San Antonio, TX: The Psychological Corporation; 2006.

11. Vincer MJ, Cake H, Graven M, Dodds L, McHugh S, Fraboni T. A population-based study to determine the performance of the cognitive adaptive test/clinical linguistic and auditory milestone scale to predict the mental developmental index at 18 months on the Bayley scales of infant development-II in very preterm infants. Pediatrics 2005;116(6):e864-7.
12. Synnes A, Luu TM, Moddemann D, et al. Determinants of developmental outcomes in a very preterm Canadian cohort. Arch Dis Child Fetal Neonatal Ed 2017;102(3):F235-F234

13. Statistics Canada. Table 051-0004 components of population growth, Canada, provinces and territories. http://www.statcan.gc.ca/tables-tableaux/sum-som/101/ cst01/demo04a-eng.htm. Updated 2016. Accessed August 4, 2017.

14. Wu YW, Lynch JK, Nelson KB. Perinatal arterial stroke: Understanding mechanisms and outcomes. Semin Neurol 2005;25(4):424-34.

15. Grunt S, Mazenauer L, Buerki SE, et al. Incidence and outcomes of symptomatic neonatal arterial ischemic stroke. Pediatrics 2015;135(5):e1220-8.

16. deVeber GA, Kirton A, Booth FA, et al. Epidemiology and outcomes of arterial ischemic stroke in children: The Canadian pediatric ischemic stroke registry. Pediatr Neurol 2017;69:58-70.

17. Heller C, Heinecke A, Junker R, et al.; Childhood Stroke Study Group. Cerebral venous thrombosis in children: A multifactorial origin. Circulation 2003;108(11):1362-7.

18. Berfelo FJ, Kersbergen KJ, van Ommen $\mathrm{CH}$, et al. Neonatal cerebral sinovenous thrombosis from symptom to outcome. Stroke 2010;41(7):1382-8.

19. Armstrong-Wells J, Johnston SC, Wu YW, Sidney S, Fullerton HJ. Prevalence and predictors of perinatal hemorrhagic stroke: Results from the kaiser pediatric stroke study. Pediatrics 2009;123(3):823-8.

20. Cole L, Dewey D, Letourneau N, et al. Clinical characteristics, risk factors, and outcomes associated with neonatal hemorrhagic stroke: A population-based case-control study. JAMA Pediatr 2017;171(3):230-8.

21. Kirton A, Armstrong-Wells J, Chang T, et al.; International Pediatric Stroke Study Investigators. Symptomatic neonatal arterial ischemic stroke: The international pediatric stroke study. Pediatrics 2011;128(6):e1402-10.

22. Husson B, Hertz-Pannier L, Renaud C, et al.; AVCnn Group. Motor outcomes after neonatal arterial ischemic stroke related to early MRI data in a prospective study. Pediatrics 2010;126(4):912-8.

23. Rutherford MA, Ramenghi LA, Cowan FM. Neonatal stroke. Arch Dis Child Fetal Neonatal Ed. 2011;97:F377-84.

24. Golomb MR, Garg BP, Saha C, Azzouz F, Williams LS. Cerebral palsy after perinatal arterial ischemic stroke. J Child Neurol 2008;23(3):279-86.

25. Wusthoff CJ, Kessler SK, Vossough A, et al. Risk of later seizure after perinatal arterial ischemic stroke: A prospective cohort study. Pediatrics 2011;127(6):e1550-7.

26. Curtis C, Mineyko A, Massicotte $P$, et al. Thrombophilia risk is not increased in children after perinatal stroke. Blood 2017;129(20):2793-800.

27. Miller V. Neonatal cerebral infarction. Semin Pediatr Neurol 2000;7(4):278-88.

28. van Buuren LM, van der Aa NE, Dekker HC, et al. Cognitive outcome in childhood after unilateral perinatal brain injury. Dev Med Child Neurol 2013;55(10):934-40.

29. Westmacott R, MacGregor D, Askalan R, deVeber G. Late emergence of cognitive deficits after unilateral neonatal stroke. Stroke 2009;40(6):2012-9.

30. Venkatesan C, Millichap JJ, Krueger JM, et al. Epilepsy following neonatal seizures secondary to hemorrhagic stroke in term neonates. J Child Neurol 2016;31(5):547-52.

31. Moharir MD, Shroff M, Pontigon AM, et al. A prospective outcome study of neonatal cerebral sinovenous thrombosis. J Child Neurol 2011;26(9):1137-44.

32. Fitzgerald KC, Williams LS, Garg BP, Carvalho KS, Golomb MR. Cerebral sinovenous thrombosis in the neonate. Arch Neurol 2006;63(3):405-9.

33. Kirton A. Advancing non-invasive neuromodulation clinical trials in children Lessons from perinatal stroke. Eur J Paediatr Neurol 2017;21(1):75-103.

34. Tanaka E, Ogawa Y, Mukai T, et al. Dose-dependent effect of intravenous administration of human umbilical cord-derived mesenchymal stem cells in neonatal stroke mice. Front Neurol 2018;9:133. 